\title{
Educación Estadística en el contexto de la sostenibilidad: una perspectiva de formación con futuros profesores de Educación Primaria
}

Statistics Education in the Context of Sustainability: A Training Perspective with Future Primary School Teachers

Educação Estatística no Contexto da Sustentabilidade: Uma Perspectiva de Formação com Futuros Professores do Ensino Primário

Israel García-Alonso Universidad de La Laguna San Cristóbal de La Laguna, España

E-mail:igarcial@ull.edu.es Orcid: 0000-0002-1158-086X

Claudia Vásquez Pontificia Universidad Católica de Chile Villarrica, Chile

E-mail:cavasque@uc.cl Orcid: 0000-0002-5056-5208 
Resumen: Hoy el profesorado de los distintos niveles educativos se ve desafiado a alfabetizar en sostenibilidad a sus estudiantes desde temprana edad. Para abordar este reto la Educación Estocástica se constituye como una herramienta que permite tender un puente hacia la Educación para el Desarrollo Sostenible. Desde este enfoque, en este trabajo se nos centramos en explorar y describir cómo futuros profesores de Educación Primaria se enfrentan al análisis y al rediseño de una tarea que fomenta el aprendizaje de estadística en estudiantes de Educación Primaria con foco en sostenibilidad. Los resultados muestran que los futuros profesores necesitan profundizar en el conocimiento estadístico que subyace en las tareas propuestas para llevar al aula. Sin embargo, cuando analizan o rediseñan actividades estadísticas con foco en sostenibilidad, los docentes sin formación específica previa son capaces de promover aspectos que redirigen la mirada hacia tareas potentes que aglutinen los contextos de los Objetivos de Desarrollo Sostenible e incorporen tanto una alta demanda cognitiva como una articulación adecuada de estos contextos a lo largo de toda la propuesta.

Palabras clave: Educación Estocástica. Alfabetizar en sostenibilidad. Educación Primaria.

Abstract: Today, teachers at different educational levels are challenged to teach sustainability literacy to their students from an early age. To address this challenge, Stochastic Education is a tool to bridge the gap towards Education for Sustainable Development. From this approach, in this paper we focus on exploring and describing how future Primary School teachers face the analysis and redesign of a task that fosters the learning of statistics in Primary School students with a focus on sustainability. The results show that prospective teachers need to deepen the statistical knowledge that underlies the proposed tasks to bring to the classroom. However, when analyzing or redesigning statistical activities with a focus on sustainability, teachers without specific prior training are able to promote aspects that redirect the gaze towards powerful tasks that bring together the contexts of the Sustainable Development Goals and incorporate both a high cognitive demand and an adequate articulation of these contexts throughout the entire proposal.

Key words: Stochastic Education. Literacy in sustainability. Primary Education.

Resumo: Hoje em dia, os professores de diferentes níveis de ensino são desafiados a ensinar a literacia da sustentabilidade aos seus alunos desde a mais tenra idade. Para enfrentar este desafio, a Educação Estocástica é um instrumento para colmatar a lacuna em direcção à Educação para o Desenvolvimento Sustentável. A partir desta abordagem, neste documento concentramo-nos em explorar e descrever como os futuros professores do Ensino Primário enfrentam a análise e redesenho de uma tarefa que fomenta a aprendizagem de estatísticas nos alunos do Ensino Primário com enfoque na sustentabilidade. Os resultados mostram que os futuros professores precisam de aprofundar o conhecimento estatístico que está subjacente às tarefas propostas para trazer para a sala de aula. Contudo, ao analisar ou redesenhar actividades estatísticas com enfoque na sustentabilidade, os professores sem formação prévia específica são capazes de promover aspectos que redireccionam o olhar para tarefas poderosas que reúnem os contextos dos Objectivos de Desenvolvimento Sustentável e incorporam tanto uma elevada procura cognitiva como uma articulação adequada destes contextos ao longo de toda a proposta.

Palavras-chave: Educação Estocástica. A alfabetização na sustentabilidade. Ensino Primário.

Recebido em 29/07/2021.

Aceito em 18/10/2021 


\section{INTRODUCCIÓN}

Desde hace varias décadas son diversos los organismos a nivel mundial que se han preocupado por impulsar acciones prácticas que nos lleven a construir juntos un futuro mejor, a través de la superación de diversos aspectos que nos aquejan (sociales, sanitarios, medioambientales, económicos, etc.) y de este modo contribuir a la protección de los derechos humanos y del planeta (UNESCO, 2015). En tal dirección la Organización de las Naciones Unidas (ONU) plantea abordar problemáticas diversas provenientes de los 17 Objetivos de Desarrollo Sostenible (ODS) (UNESCO, 2017) que abarcan tres grandes sectores que reclaman atención: económico, social y ambiental, y que permitirían implementar una Educación para el Desarrollo Sostenible (EDS) que permee en los distintos niveles educativos. Para ello, de acuerdo con la UNESCO (2017), se requiere de una educación holística, integradora y transformadora, centrada en: a) los contenidos y los resultados de aprendizaje; b) la pedagogía y entornos de aprendizaje; c) los frutos del aprendizaje; y d) la transformación social. Esto sin duda es un nuevo reto para los profesores y los formadores de profesores, y más aún en las áreas de física, química y matemáticas (Uitto \& Saloranta, 2017; Dahl, 2019; Vásquez et al., 2020; Vásquez et al., 2021).

Por consiguiente, hay que brindar oportunidades de desarrollo profesional al profesorado en formación y en ejercicio, de manera que cuenten con las herramientas necesarias para incluir la EDS en las distintas disciplinas escolares (Vilches \& Gil, 2012). Pues no denbemos olvidar que el profesorado es una gente de cambio poderoso, y son finalmente ellos los llamados a llevar la sostenibilidad al aula escolar, y de favorecer el desarrollo de las competencias clave necesarias para fomentar el desarrollo sostenible (Calero et al., 2019). A este respecto, la UNESCO (2017) subraya que no se trata de "sólo integrar contenidos como el cambio climático, la pobreza y el consumo sostenible [..] sino también crear contextos de enseñanza y aprendizaje interactivos y centrados en el alumno" (p.7).

En este sentido, "la Educación Estocástica es un terreno fértil para ayudar a crear conciencia, comprender, reflexionar y actuar en torno a uno de los desafíos más 
apremiantes del mundo actual: la Educación para el Desarrollo Sostenible" (Vásquez, 2020, p. 15). Desde este posicionamiento, Vásquez (2020, 2021), Vásquez y GarcíaAlonso (2020) y Vásquez y Alsina (2021) proponen una Educación Estocástica para Educar en Sostenibilidad, cuyo propósito es favorecer el desarrollo de la alfabetización estadística y probabilística (Gal,2002, 2005), así como de conocimientos, competencias, valores y actitudes con los que contribuyan al desarrollo sostenible, en pos de tomar decisiones para crear un mundo más sostenible.

Desde esta perspectiva, en el marco del Enfoque de la Educación Estocástica para Educar en Sostenibilidad, en este estudio nos centramos en explorar y describir cómo futuros profesores de Educación Primaria se enfrentan al análisis y al rediseño de una tarea que fomenta el aprendizaje de estadística en estudiantes de Educación Primaria con foco en sostenibilidad.

\section{EDUCACIÓN ESTOCÁSTICA UNA HERRAMIENTA PARA EDUCAR EN SOSTENIBILIDAD}

Cada vez es más necesario que todos los ciudadanos adquieran oportunamente las habilidades y conocimientos que les permita desempeñarse como ciudadanos críticos, capaces de construir sociedades cada vez más democráticas (e.g. Wild et al., 2018; Batanero, 2019; Engel, 2019; Vásquez, 2020). Por su parte, el conocimiento Estadístico y Probabilístico tiene por objetivo desarrollar ciudadanos capaces de comprender, evaluar y razonar a partir de la interpretación de datos e informaciones estadísticas (Gal 2002, 2005), así como lograr las habilidades y conocimientos necesarios para establecer conexiones entre problemáticas provenientes de diversos contextos (Batanero \& Borovcnik, 2016; Ben-Zvi et al., 2018). Este conocimiento enlaza con el desafío de contar con ciudadanos alfabetizados en sostenibilidad, es decir, ciudadanos que más allá de poseer conocimientos sobre sostenibilidad, cuenten con las habilidades, actitudes, competencias, disposiciones y valores necesarios para avanzar en pos de frenar y superar el actual deterioro que aqueja a nuestro mundo (Stibbe, 2014). Así, esta amalgama de conocimientos y competencias asociadas a la 


\section{Universidade Federal da Grande Dourados}

toma de decisiones presente en el conocimiento estocástico y de la EDS se hace desde el contexto y vuelve a él, como medio de acción y conocimiento. En este sentido, con la implementación del conocimiento de la Estadística desde la perspectiva crítica logramos "que el estudiante ponga todo ese conocimiento disciplinar y las habilidades adquiridas en los procesos investigativos al servicio de la formación de su consciencia social y de la transformación de la sociedad" (ZapataCardona, 2018, p. 35), lo que enlaza perfectamente con la EDS.

Por tanto, utilizamos el conocimiento estocástico para promover la EDS, pues "son números en contexto" (Coob \& Moore, 1997), cuya enseñanza parte de contextos reales, con significado para los estudiantes y que se encuentren adaptados a su edad y etapa escolar, y que desarrolla en los estudiantes la argumentación, la formulación de conjeturas y la reflexión, de forma que "lo que importa no son los datos, sino las respuestas y los conocimientos que buscamos en los datos" (Gal, 2019, p. 4). Pero, debemos saber que el contexto "no está presente automáticamente en el aula tenemos que introducirlo" (Gal, 2019, p. 3), lo que se traduce en una mayor responsabilidad de los docentes quienes finalmente serán los encargados de realizar la selección y adaptación de los contextos que sean más adecuados al aula escolar y que favorezcan a la vez el desarrollo de la alfabetización estadística.

Pero ¿qué contextos utilizar?, de manera que tales contextos favorezcan el desarrollo de competencias para conocer y actuar ante los desafíos del mundo actual. Se sabe que el contexto en estadística y probabilidad toma especial relevancia en el análisis de los aspectos sociopolíticos (Frankenstein, 2009; Lesser, 2007) y el caso de la Educación Estocástica para educar en sostenibilidad se acerca a este ámbito. Así, Pfannkuch y Wild (2004) indican la necesidad de resolver problemas del mundo real para desarrollar en los estudiantes el pensamiento estadístico, pues contribuye a realizar predicciones, buscar explicaciones, encontrar las causas dentro de contextos específicos. Y, tanto Garfield (2002) como Ben-Zvi y Garfield (2004), indican que es necesario realizar interpretaciones basadas en conjuntos de datos, representación de 


\section{Universidade Federal da Grande Dourados}

datos o resúmenes estadísticos de datos cuando se desarrolla el razonamiento estadístico.

Llegados a este punto, observamos que los ODS son una fuente de contextos que pueden ser de interés en los distintos niveles educativos (Vásquez \& García-Alonso, 2020). Y, en este sentido, encontramos que la formación en Educación Estocástica es un buen instrumento para el desarrollo de competencias vinculadas a la EDS (Vásquez, 2020; Vásquez \& García-Alonso, 2020; Vásquez \& Alsina, 2021), en la que "los conceptos estadísticos vinculados al contexto deben abordarse como construcciones sociales, siguiendo la estrategia sugerida por el aprendizaje estadístico orientado a los datos" (Gattuso \& Ottaviani, 2011, p. 124).

Desde este prisma, conscientes del desafio de diseñar procesos de enseñanza y aprendizaje que respondan a las necesidades actuales, se hace evidente la necesidad de repensar la enseñanza de la estocástica en el aula escolar, de manera tal que se transite hacia una Educación Estocástica para Educar en Sostenibilidad (Vásquez, 2020). Pero, esta reorientación no solo implica que los estudiantes comprendan las distintas problemáticas (sociales, económicas y medioambientales), sino que es necesario ir más allá, de manera tal que los estudiantes tomen conciencia de estas problemáticas y actúen para avanzar hacia un mundo más sostenible (UNESCO, 2017). Desde este enfoque, Vásquez y García-Alonso (2020), plantean la necesidad de que el profesorado diseñe e implemente tareas de estadística y probabilidad con foco en sostenibilidad, las cuales deben resguardar diversas características que se ilustran en la Figura 1.

Cada una de las características anteriores, aborda el análisis de las tareas con foco en diferentes aspectos, con el objeto de tener un análisis exhaustivo que permita indicar si la tarea ofrece un enfoque cercano a la EDS. 


\section{Universidade Federal da Grande Dourados}

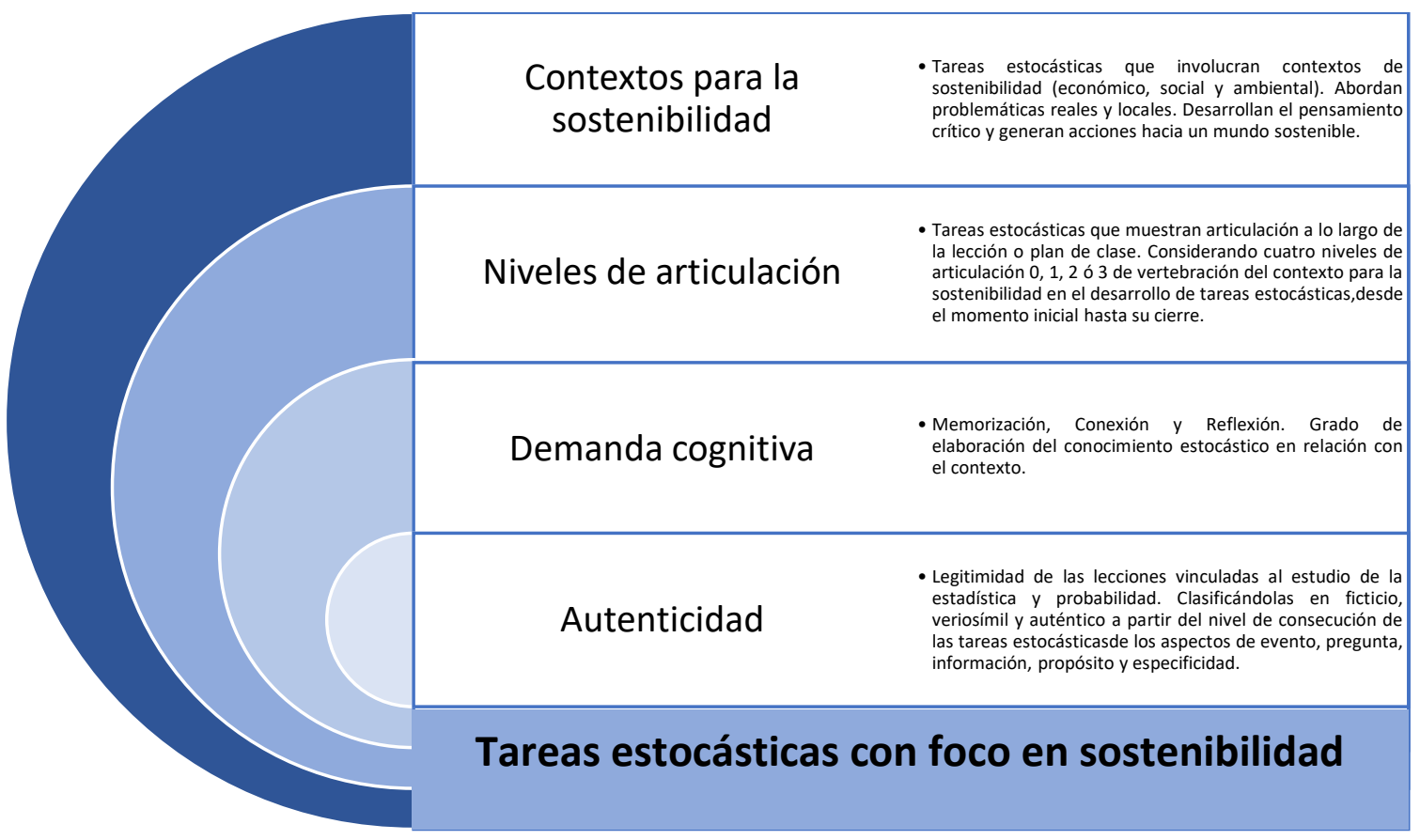

Figura 1. Aspectos por considerar en el diseño de tareas estocásticas con foco en sostenibilidad.

Fuente: elaboración propia.

Nos encontramos con una herramienta cuya única finalidad no es sólo describir la tarea o lección, sino que además orienta sobre los aspectos que debemos incorporar, de forma que "estas características se convierten en una hoja de ruta para el profesorado indicando las características a incorporar en el diseño de tareas estocásticas enfocadas a educar en sostenibilidad promoviendo la acción en su entorno" (Vásquez et al., 2021, p.18).

\section{Metodología}

En este estudio se desarrolla un trabajo teórico y cualitativo (McMillan \& Schumacher, 2001), en el que utilizamos el análisis del contenido como técnica (Stemler, 2001), pues el propósito es explorar y describir el análisis que realizan futuros profesores de Educación Primaria de tareas estadísticas con foco en 
sostenibilidad extraídas de Vásquez y García-Alonso (2020); así como el rediseño que realizan de ellas.

\section{Participantes y contexto}

En este estudio han participado 124 estudiantes del Grado de Maestro en Educación Primaria de España. Estos estudiantes se encuentran en 3o curso del citado grado y han recibido una formación didáctica y disciplinar en la materia de Didáctica de la Numeración, de la Estadística y del Azar. Dicha formación se ha centrado en los aspectos relacionados con la numeración en la Educación Primaria, y poseen un conocimiento de los aspectos que deben tener en cuenta como futuros profesores cuando inician la enseñanza de conceptos y procedimientos matemáticos en el aula. Sobre el conocimiento y enseñanza de la estadística, no han recibido formación con anterioridad.

\section{Desarrollo de la actividad (intervención didáctica)}

Se realizó se realizó una intervención didáctica a los estudiantes antes descritos durante el primer semestre académico de 2020/21. Dicha intervención consideró las etapas que se señala en la Figura 2.

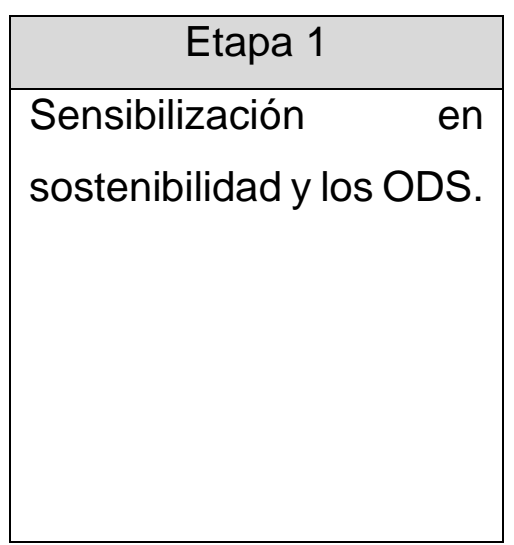

\begin{tabular}{|l|}
\hline \multicolumn{2}{|c|}{ Etapa 2} \\
\hline Análisis/evaluación de \\
las tareas ES-2 y ES-5 \\
de estadística con foco \\
en sostenibilidad \\
extraídas de Vásquez y \\
García-Alonso (2020). \\
\hline
\end{tabular}

\begin{tabular}{|l|}
\hline Etapa 3 \\
\hline Rediseño de las tareas \\
de estadísticas con foco \\
en sostenibilidad. \\
\end{tabular}

Figura 2. Etapas de la intervención didáctica.

Fuente: elaboración propia.

En la etapa 1, al igual que en el estudio de Vásquez y García-Alonso (2020), los futuros profesores han sido sensibilizados en relación con los ODS y la Educación para la Sostenibilidad, con objeto de que tomen conciencia acerca de la importancia 


\section{Universidade Federal da Grande Dourados}

de incorporarlo en el aula escolar. Las actividades desarrolladas en torno a la EDS han considerado una sesión de 2 horas de duración acompañada de análisis documental y audiovisual, así como el análisis de libros de texto y proyectos desarrollados en el aula, ninguno de ellos en relación con la estadística y la probabilidad. Luego, se organizó a los estudiantes en grupos de 4, obteniendo en total 31 grupos. Así, en la etapa 2, se proporcionó a los grupos las tareas ES-2 (Figura 3) y ES-5 (Figura 4) de estadística con foco en sostenibilidad extraídas de Vásquez y García-Alonso (2020), las cuales en dicho estudio fueron categorizadas, dentro de la autenticidad, como tareas verosímil y auténtica, respectivamente.

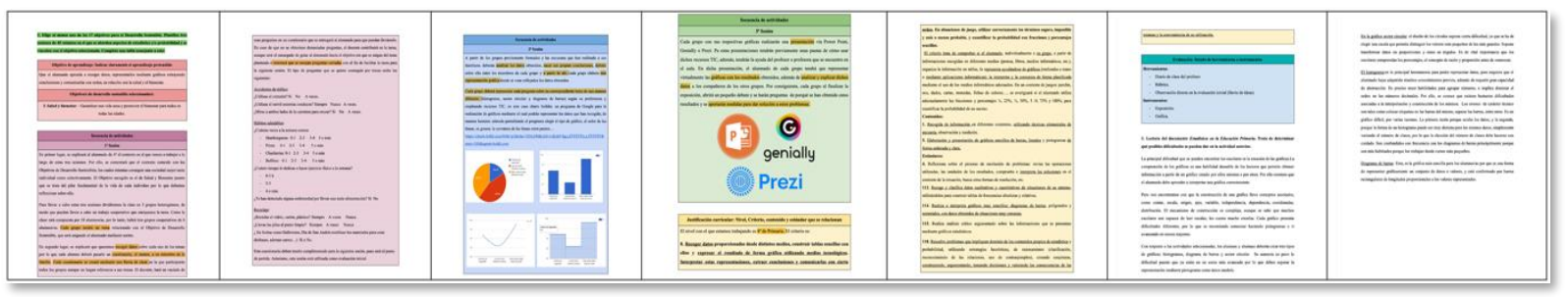

Figura 3. Tarea ES-2 proporcionada a los futuros profesores.

Fuente: elaboración propia.

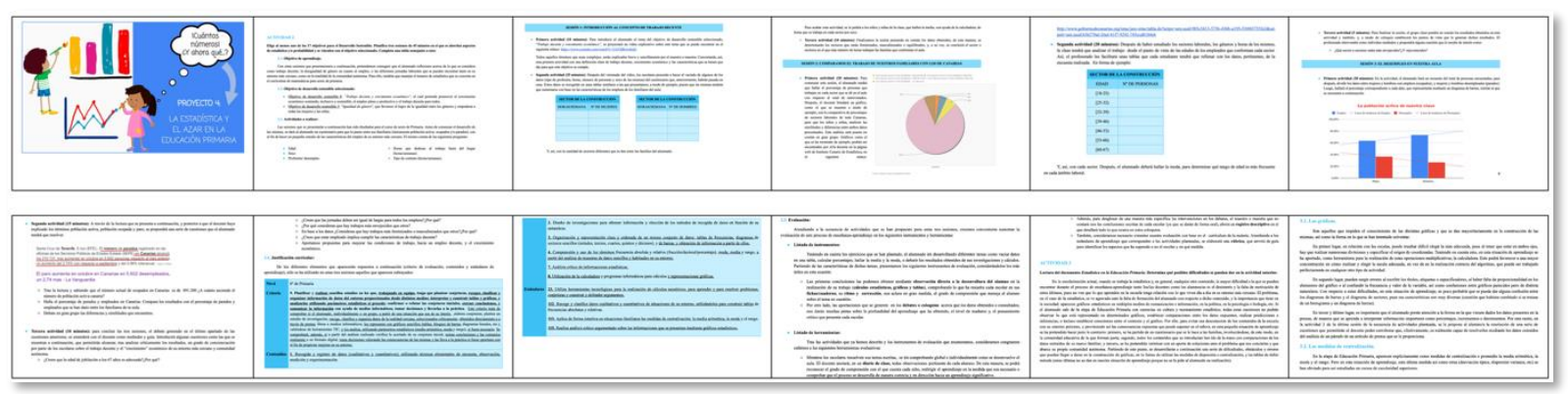

Figura 4. Tarea ES-5 proporcionada a los futuros profesores.

Fuente: elaboración propia.

Para llevar a cabo la actividad, a 15 grupos se les ofrece la propuesta verosímil (ES-2) y a los otros 16 grupos la propuesta auténtica (ES-5). A partir de tales diseños de unidades didácticas, se les solicitó estudiar la propuesta asignada y elaborar un 


\section{Universidade Federal da Grande Dourados}

informe que evalúe dicha propuesta atendiendo a la coherencia que presenta entre los ODS seleccionados, las actividades sugeridas y la evaluación presentada. Finalmente, en la etapa 3, partiendo de la evaluación anterior, debieron rediseñar tales propuestas de unidades didácticas, señalando sus propias sugerencias de mejora de forma que logren un mejor desarrollo de la EDS.

\section{Categorías para el análisis}

El análisis de las respuestas de los estudiantes se ha realizado a partir de las cuatro categorías de estudio propuestas por Vásquez et al. (2021) para analizar el diseño de actividades estadísticas con foco en la sostenibilidad (Figura 1):

a) Contextos para la sostenibilidad: se observan los tipos de contextos (económico, social y ambiental) que están presentes en la enseñanza de la estadística y la probabilidad. Se abordan problemáticas provenientes de contextos reales y locales, que impulsan el desarrollo de un pensamiento crítico, permitiendo a los estudiantes comprender y reflexionar sobre la realidad, y la importancia de contribuir desde las propias acciones al desarrollo de un mundo sostenible. Además, se consideraron las categorías: "otros contextos no significativos para la sostenibilidad", que incluye los contextos que no se vinculan con los ámbitos de acción de la EDS; y "sin contexto", donde se incluyen tareas que no consideran ningún tipo de contexto.

b) Niveles de articulación: se observa el grado en el que se desarrolla cada contexto para la sostenibilidad, a partir de los siguientes cuatro niveles:

- Nivel 0: el contexto que se presenta inicialmente no hace referencia a ninguno de los contextos para la sostenibilidad.

- Nivel 1: el contexto para la sostenibilidad aparece en una actividad inicial o motivacional, pero no se recurre en ninguna otra tarea a él.

- Nivel 2: el contexto para la sostenibilidad aparece de forma discontinua a lo largo de la lección y no vertebra todas las tareas que se proponen. 
- Nivel 3: el contexto para la sostenibilidad vertebra toda la lección propuesta, desde el momento inicial hasta su cierre.

c) Demanda cognitiva: se observa en qué medida la tarea propuesta requiere un conocimiento más elaborado acerca del conocimiento estadístico y probabilístico y su relación con el contexto de trabajo. Se utiliza la taxonomía para el análisis de la demanda cognitiva de las tareas matemáticas propuesta por el NCTM (2014). De acuerdo con esta taxonomía, se diferencian los siguientes tres niveles de demanda cognitiva según se relaciona con el conocimiento estadístico y/o probabilístico con el contexto y las acciones que se deben desarrollar en la actividad:

- Memorización: el foco de la tarea está en la reproducción memorística de aprendizajes previos; construcción de tablas/gráficos a partir de datos ofrecidos; y/o identificación de elementos que la forman (procedimiento de construcción), sin modificación de la información contenida.

- Conexión: se focaliza en establecer conexiones entre conceptos estadísticos y probabilísticos, que requieren un conocimiento amplio y a la vez conectar diferentes representaciones para la comprensión de la estadística y/o la probabilidad. Pero el contexto no tiene un papel fundamental o no lleva al planteamiento de nuevas preguntas con objeto de un mayor conocimiento estadístico y/o probabilístico.

- Reflexión: requieren la elaboración de conclusiones conectadas con los EDS. En este tipo de tareas, el contexto se convierte en fuente de aprendizaje estadístico y/o probabilístico y fuente de reflexión sobre el rol individual en la comunidad local y en la sociedad en la que participa.

d) Autenticidad: se observa la legitimidad de las lecciones vinculadas al estudio de la estadística y la probabilidad, a partir de una adaptación de los indicadores propuestos por Chamoso y Cáceres (2018): 


\section{Universidade Federal da Grande Dourados}

- Evento: la situación planteada es factible en la vida real fuera de la escuela y considera contextos para la sostenibilidad (datos cercanos al entorno de la localidad o del país o cercanos a la situación del contexto donde viven).

- Pregunta: se formula de manera habitual para el evento descrito y cuya respuesta tiene un valor estadístico y/o probabilístico práctico o es interesante por el debate que suscita.

- Información: los datos coinciden con los reales o se han adecuado para resaltar el contexto para la sostenibilidad abordado. Los datos que se utilizan en la tarea son datos conseguidos por medio de encuestas, son datos reales o bien adecuados para la edad y nivel en el que se proponen.

- Propósito: se menciona explícitamente y está en consonancia con el planteado desde la situación real y algún contexto para la sostenibilidad. La tarea desarrollada lo hace de forma que se dirige a abordar el contexto para la sostenibilidad planteado y el contenido de estadística y/o probabilidad contribuye de forma decisiva en su consecución.

- Especificidad de los datos: los datos son recogidos a partir de encuestas y/o experimentos realizados por los estudiantes o se menciona la procedencia de los datos o gráficos con los que trabajan. Tales datos son adecuados para dar respuesta a la pregunta planteada o bien encaminan a los estudiantes para que alcancen las conclusiones que se pretenden.

De acuerdo con Paredes et al., (2020), a partir de este enfoque de autenticidad, una tarea puede considerarse auténtica, verosímil o ficticia, dependiendo de los aspectos de autenticidad que verifique. Así, se considerará una tarea verosímil cuando verifica los indicadores de autenticidad descritos como "evento", "pregunta" e "información". La tarea se considerará auténtica si verifica todos los aspectos de la autenticidad. 


\section{Universidade Federal da Grande Dourados}

\section{Procedimiento de análisis}

El procedimiento seguido parte del estudio del informe de evaluación y análisis hecho por los futuros profesores a las tareas ES-2 (Figura 3) y ES-5 (Figura 4), catalogadas como Verosímil y Auténtica en Vásquez y García-Alonso (2020), respectivamente.

Para llevar a cabo el análisis dividimos las categorías anteriores según el producto que han elaborado los estudiantes en cada etapa del estudio. Así, para la etapa 1, en la que el producto es un informe, realizamos el análisis a través de las categorías contexto y nivel de articulación. Es decir, que observamos qué opinión merece para los estudiantes el contexto que se presenta en las tareas ES-2 y ES-5, y si consideran que estos contextos se encuentran bien articulados.

La etapa 2 consiste en la elaboración y propuesta de nuevas actividades que parten del informe anteriormente elaborado. Estas actividades formarán parte del rediseño que elaboran los futuros profesores y, por tanto, nos pueden dar información sobre la demanda cognitiva que presentará el rediseño y, en el caso del ES-2, acerca de la autenticidad. La razón por la que sólo tomamos las nuevas actividades propuestas para estudiar la autenticidad es que esta tarea es considerada verosímil, con lo cual tiene algunas características que se pueden mejorar. Serán estas características las que centrarán la atención de la investigación y nos permitirá conocer si las cubren las actividades del rediseño.

\section{Resultados}

Con el propósito de explorar y describir cómo futuros profesores de Educación Primaria se enfrentan al análisis y al rediseño de una tarea que fomenta el aprendizaje de estadística en estudiantes de Educación Primaria con foco en sostenibilidad, los resultados se han organizados en dos subapartados que dan cuenta, en primer lugar, del análisis sobre el informe elaborado por los futuros profesores; y, en segundo lugar, el análisis del rediseño propuesto. 
A. Análisis del informe elaborado acerca de las propuestas analizadas

Los estudiantes debían realizar un informe sobre los elementos que observaban al revisar las propuestas ES-2 (Figura 3) y ES-5 (Figura 4) (Vásquez y García-Alonso, 2020). Los elementos analizados en dichos informes nos dan información acerca de las categorías: contexto y niveles de articulación.

\section{Contexto}

La propuesta ES-2 (Figura 5) se centra en el ODS "Garantizar una vida sana y promover el bienestar para todos en todas las edades" (ODS-3), mientras que la propuesta ES-5 (Figura 6) desarrolla dos ODS, "Trabajo decente y crecimiento económico" (ODS-8) e "Igualdad de Género" (ODS-5). Cada uno de estos ODS determina el contexto de la propuesta en la que se desarrollará el estudio estadístico y que justificarán las acciones de mejora que se proponen.

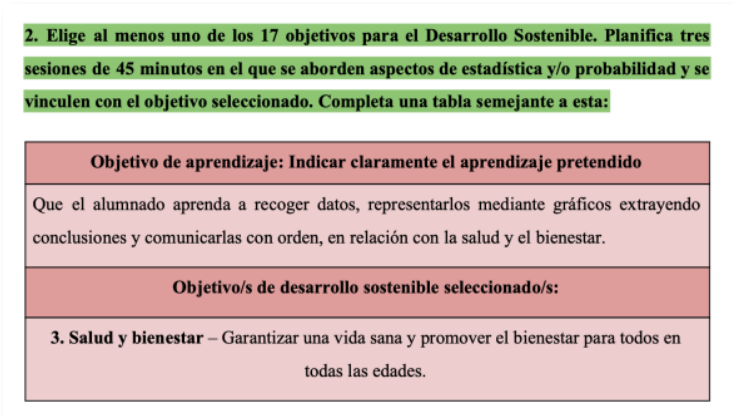

Figura 5. Objetivos propuesta ES-2.

Fuente: Vásquez y García-Alonso (2020).

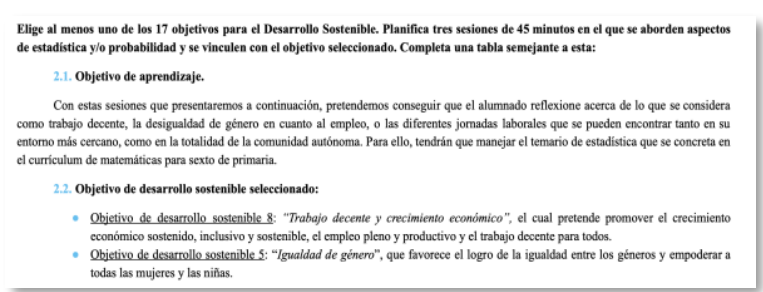

Figura 6. Objetivos propuesta ES-5. Fuente: Vásquez y García-Alonso (2020).

Con respecto al contexto, observamos que los futuros profesores consideraron en algunos casos que no se ajustaba correctamente el contexto con las actividades que luego se proponen. En el informe elaborado por los futuros docentes hemos observado que se dieron cuatro tipos de respuesta principales: unos grupos consideran que el contexto es adecuado, otros que es mejorable y hacen sugerencias de alguna modificación, y hay grupos que no lo consideran adecuado. También hay algún grupo que no hace ningún comentario acerca del contexto (Tabla 1). 


\section{Tabla 1}

Categorías de respuestas acerca del contexto del ODS.

\begin{tabular}{rcc}
\cline { 2 - 3 } & ES-2 & ES-5 \\
\hline Contexto adecuado y desarrolla el ODS & 0 & $5(31.2 \%)$ \\
\hline Contexto mejorable: sugieren modificaciones & $10(66.7 \%)$ & $4(25 \%)$ \\
\hline Contexto no adecuado & $5(33.3 \%)$ & $5(31.2 \%)$ \\
\hline No se hace ningún comentario & 0 & $2(12.6 \%)$ \\
\hline Total & $\mathbf{1 5}$ & $\mathbf{1 6}$ \\
\hline
\end{tabular}

Fuente: Elaboración propia

A partir de la Tabla 1 observamos, por un lado, en el análisis del ES-2 todos los equipos están de acuerdo con que se lleven a cabo modificaciones en torno al contexto. Mientras que, los grupos que analizaron el ES- 5 sugieren cambios poco más de la mitad de los grupos. Y sólo en el análisis de la propuesta ES- 5 encontramos 5 grupos que consideran adecuado el contexto que se ha seleccionado en la propuesta.

Por su parte, en la Figura 7 se muestran dos ejemplos de respuestas, pertenecientes a cada propuesta, en las que se plantea que las actividades "no se enfocan" o bien están "muy en el aire", haciendo ver que el contexto que se propone para desarrollar cada uno de los ODS requieren de mayor ajuste. Además, en el primer caso se ofrecen contextos a modo de ejemplo, mientras que en el segundo ejemplo se sugiere un nivel mayor de concreción de los ODS de forma que estas pequeñas metas u sub-objetivos hagan más abordable la propuesta.

\footnotetext{
$\rightarrow$ Atendiendo a la relación entre el ODS y el marco curricular:

- Durante la mayor parte del proyecto no se trabaja como tal el objetivo de desarrollo sostenible, puesto que únicamente se plantea como tema para realizar las actividedes, sin enfocarse en plantear/ trabajar con dicho objetivo, ya sea, indicando a los alumnos que planteen hábitos de vida saludable, construyendo una dieta equilibrada,

Respuesta grupo 101-02 sobre ES-2
}

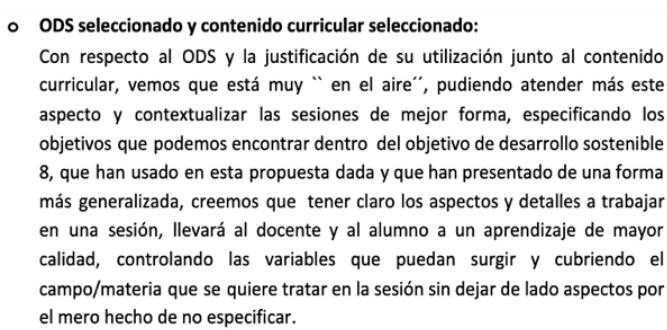
aspecto y contextualizar las sesiones de mejor forma, especificando los objetivos que podemos encontrar dentro del objetivo de desarrollo sostenible 8 , que han usado en esta propuesta dada y que han presentado de una forma más generalizada, creemos que tener claro los aspectos y detalles a trabajar en una sesión, llevará al docente y al alumno a un aprendizaje de mayor calidad, controlando las variables que puedan surgir y cubriendo el campo/materia que se quiere tratar en la sesión sin dejar de lado aspectos por el mero hecho de no especificar.

Respuesta grupo 101-17 sobre ES-5

Figura 7. Respuestas de dos grupos en la categoría "Contexto mejorable". 


\section{Universidade Federal da Grande Dourados}

Fuente: Elaboración propia.

\section{Niveles de articulación}

La articulación de las actividades en una propuesta didáctica se observa a través del análisis del desarrollo del contexto en las diferentes actividades propuestas. Así, al analizar ambas propuestas (Figura 8), se observa que la propuesta ES-2 se propone, como arranque, recoger datos mediante una encuesta, acerca del tema del ODS seleccionado, luego se lleva a cabo el análisis de estos datos y finaliza con la presentación de los resultados al resto de la clase. Mientras que la propuesta ES-5 parte de una sesión de sensibilización sobre el concepto de trabajo decente, continúa con una recogida de datos en el entorno sobre el tipo de trabajo que realizan sus familiares, y concluyen con una actividad de comparación entre el desempleo de las familias estudiadas y los datos oficiales sobre ello, lo que dará pie a un pequeño debate sobre estas cuestiones.

En segundo lugar, se explicará que queremos recoger datos sobre cada uno de los tema por lo que cada alumno deberá pasarle un cuestionario, al menos, a un miembro de la familia. Cada cuestionario se creará mediante una lluvia de ideas en la que participará todos los grupos aunque no hagan referencia a sus temas. El docente, hará un vaciado de

A partir de los grupos previamente formados $y$ las encuestas que han realizado a sus familiares deberán analizar los datos obtenidos, sacar sus propias conclusiones, debatir sobre ello entre los miembros de cada grupo y a partir de ahí, cada grupo elabora una representación gráfica donde se vean reflejados los datos obtenidos.

Cada grupo con sus respectivas gráficas realizarán una presentación vía Power Point, Genially o Prezi. Pa estas presentaciones tendrán previamente unas pautas de cómo usar
SESIÓN 1: INTRODUCCIÓN AL CONCEPTO DE TRABAJO DECENTE

SESIÓN2: COMPARAMOS EL TRABAJO DE NUESTROS FAMILIARES CON LOS DE CANARIAS

SESIÓN 3: EL DESEMPLEO EN NUESTRA AULA

Propuesta ES-5

\section{Propuesta ES-2}

Figura 8. Parte de las actividades que promueve cada propuesta analizada.

Fuente: Vásquez y García-Alonso (2020). 


\section{Universidade Federal da Grande Dourados}

En las respuestas dadas por los distintos grupos tras su análisis observamos dos categorías principales: por un lado, aquellos que indican que el contexto para la sostenibilidad se articula correctamente en la propuesta y, por otro, los que consideran que no se presenta bien articulado. También encontramos algunos grupos que no hicieron ningún comentario sobre este aspecto (Tabla 2).

\section{Tabla 2}

Categorías de respuestas acerca de los niveles de articulación.

\begin{tabular}{rcc}
\cline { 2 - 3 } & ES-2 & ES-5 \\
\hline Contexto para la sostenibilidad bien articulado & $5(33.3 \%)$ & $7(43.8 \%)$ \\
\hline Contexto para la sostenibilidad no se presenta & $9(60 \%)$ & $4(25 \%)$ \\
bien articulado & & \\
\hline No comentan la articulación del contexto & $1(6.7 \%)$ & $5(31.2 \%)$ \\
\hline Total & $\mathbf{1 5}$ & $\mathbf{1 6}$ \\
\hline
\end{tabular}

Fuente: Elaboración propia.

Cabe destacar que la mayoría de los grupos que analizaron la propuesta ES-2 opinan que el contexto para la sostenibilidad no se encuentra bien articulado, mientras que los que analizaron la ES-5 o bien consideran que es correcta o bien no hacen ningún comentario al respecto.

El análisis de la articulación del contexto para la sostenibilidad se realizó a través de los comentarios que los futuros profesores hicieron con respecto a las actividades, pues interpretamos que consideran que no se articulan correctamente cuando señalan o proponen actividades diferentes que consideran que focalizan mejor la propuesta que están analizando en el contexto que se ha propuesto (Figura 9). Algunos de los grupos de estudiantes que valoraron el ES-2 consideraron poco adecuado trabajar el reciclaje para el ODS que se había seleccionado, o bien sugirieron nuevos contextos de trabajo, en los que las actividades estuvieran, según ellos, más centradas en alguno de los contextos de sostenibilidad que determina el ODS. En cambio, entre los equipos que analizaron el ES-5 hubo bastantes menos que consideraron que no se 


\section{Universidade Federal da Grande Dourados}

encontraba esta propuesta bien articulada. Entre los equipos que sugirieron que las actividades no eran adecuadas basaron esta afirmación en que la temática seleccionada en el proyecto (el trabajo decente) se aleja del interés de los estudiantes de este nivel educativo y, como consecuencia, esto limitaría el alcance de las actividades. Hubo algún equipo que sugirió que la propuesta ES-5 aborda dos ODS y no lo hace de forma equilibrada, lo que señalaban como un aspecto negativo.

- ODS seleccionado y contenido curricular seleccionado

En primer lugar, observamos que en lo referente a los ODS seleccionados, en la primera sesión, cuando realizan el modelo de encuestas, no es adecuada la que hace referencia al reciclaje, ya que ese objetivo de aprendizaje no se incluye en los temas sugeridos para el ODS 3 "Salud y Bienestar". Este se encuentra en los objetivos de aprendizaje para el ODS 11 "Ciudades y Comunidades Sostenibles": "Generación y gestión de desechos (prevención, reducción, reciclaje, reutilización)"

\section{Respuesta grupo 101-12 sobre ES-2}

Por tanto, no tiene demasiado sentido utilizar este tema con el fin de trabajar aspectos de salud y el bienestar. En todo caso, habría que ajustar el tema a lo relacionado con los químicos, la polución y la contaminación del aire, el agua y el suelo, y no tanto a la reutilización de materiales, etc.
Consideramos que algunas actividades no están enfocadas de la mejor manera; en el caso del cuestionario, estamos de acuerdo en que se realice un tipo de encuesta/cuestionario para que el alumnado encuentre el contexto de las actividades en su entorno más cercano. No obstante las preguntas propuestas nos parecen invasivas a la intimidad de cada familia.

\section{Respuesta grupo 101-05 sobre ES-5}

Otro parámetro que nos parece poco oportuno es el contexto de las preguntas realizadas en el ejercicio 3 por ejemplo. pensamos que son preguntas que salen de lo que el alumnado puede entender con su edad sobre el trabajo.

\section{Respuesta grupo 101-13 sobre ES-5}

Figura 9. Respuestas de dos grupos en la categoría "Niveles de articulación".

Fuente: Elaboración propia.

\section{B. Rediseño de la propuesta analizada}

En este apartado nos centramos en el rediseño que proponen los futuros profesores a la propuesta analizada, y en el análisis ponemos el foco en las características: demanda cognitiva y autenticidad.

Antes de entrar a analizar estas categorías, observamos que la mayor parte de los distintos grupos de estudiantes crean nuevas actividades que sustituyen 0 complementan las que ya aparecen en las propuestas ES-2 o ES-5 (Tabla 4) y muy pocos grupos sólo dan una descripción de la actividad. 
Tabla 4

Nuevas propuestas.

\begin{tabular}{rcc}
\cline { 2 - 3 } & ES-2 & ES-5 \\
\hline Actividades nuevas para una única sesión & 2 & 5 \\
\hline Actividades nuevas para más de una sesión & 10 & 9 \\
\hline Sólo describen las actividades sin especificar & 3 & 2 \\
\hline Total & $\mathbf{1 5}$ & $\mathbf{1 6}$ \\
\hline
\end{tabular}

Fuente: Elaboración propia.

\section{Demanda Cognitiva}

En las propuestas didácticas que evaluaron los futuros profesores se señaló la demanda cognitiva atendiendo al mayor nivel de demanda alcanzado al menos por una de las actividades propuestas. Fue así como la propuesta ES-2 se considera de la categoría de conexión y la propuesta ES-5 de reflexión (Figura 10), por contener una actividad, al menos de dicha demanda.

\begin{tabular}{|l|}
\hline \multicolumn{1}{|c|}{$3^{a}$ Sesión } \\
\hline Cada grupo con sus respectivas gráficas realizarán una presentación vía Power Point, \\
Genially o Prezi. Pa estas presentaciones tendrán previamente unas pautas de cómo usar \\
dichos recursos TIC, además, tendrán la ayuda del profesor o profesora que se encuentre en \\
el aula. En dicha presentación, el alumnado de cada grupo tendrá que representar \\
virtualmente las gráficas con los resultados obtenidos, además de analizar y explicar dichos \\
datos a los compañeros de los otros grupos. Por consiguiente, cada grupo al finalizar la \\
exposición, abrirá un pequeño debate y se harán preguntas de porqué se han obtenido estos \\
resultados y se aportarán medidas para dar solución a estos problemas.
\end{tabular}

Demanda de conexión (propuesta ES-2)
Tercera actividad (10 minutos): para concluir las tres sesiones, el debate generado en el último apartado de las cuestiones anteriores, se extenderá con el docente como mediador y guía. Introducirá algunas cuestiones como las que se muestran a continuación, que permitirán alcanzar, tras analizar criticamente los resultados, un grado de concienciación por parte de los escolares sobre el trabajo decente y el "crecimiento" económico de su entorno más cercano y comunidad autónoma.

- ¿Creen que la edad de jubilación a los 67 años es adecuada?:Por qué?

¿Creen que las jornadas deben ser igual de largas para todos los empleos?:Por qué?

- ¿Por qué consideran que hay trabajos más envejecidos que otros?

- En base a los datos ¿Consideras que hay trabajos más feminizados o masculinizados que otros??:Por qué?

- ¿Crees que estar empleado implica cumplir las características de trabajo decente?

- Aportamos propuestas para mejorar las condiciones de trabajo, hacia un empleo decente, y el crecimiento económico

\section{Demanda de reflexión (propuesta ES-5)}

Figura 10. Actividad de mayor demanda cognitiva de cada propuesta.

Fuente: Vásquez y García-Alonso (2020). 
En la Tabla 3 se muestra la categoría alcanzada por alguna de actividades que los estudiantes han propuesto, con objeto de determinar la demanda cognitiva que posee, según la descripción de esta categoría.

\section{Tabla 3}

Categorías de respuestas acerca del dominio cognitivo.

\begin{tabular}{rcc}
\cline { 2 - 3 } & ES-2 & ES-5 \\
\hline Reflexión: comparación y conclusión estadística & $4(26.7 \%)$ & $6(37.5 \%)$ \\
\hline Conexión: conceptos estadísticos y parámetros & $6(40 \%)$ & $5(33.3 \%)$ \\
\hline Memorización: tablas y gráficos estadísticos & $5(33.3 \%)$ & $5(33.3 \%)$ \\
\hline Total & $\mathbf{1 5}$ & $\mathbf{1 6}$
\end{tabular}

Fuente: Elaboración propia.

Observamos que, la mayoría de las respuestas, se encuentran en ambos casos en las categorías más elevadas de demanda cognitiva, siendo ligeramente superior en las actividades ofrecidas por los equipos que analizaron la propuesta ES-5. Y en ambos casos, un tercio de las respuestas sólo alcanzan la categoría de memorización.

Por otro lado, cuatro de los 15 equipos de futuros profesores se percataron de un error en una gráfica estadística que aparece en la propuesta ES-2, llevándolos a ofrecer actividades para corregir este error (Figura 11). 


\title{
Universidade Federal da Grande Dourados
}

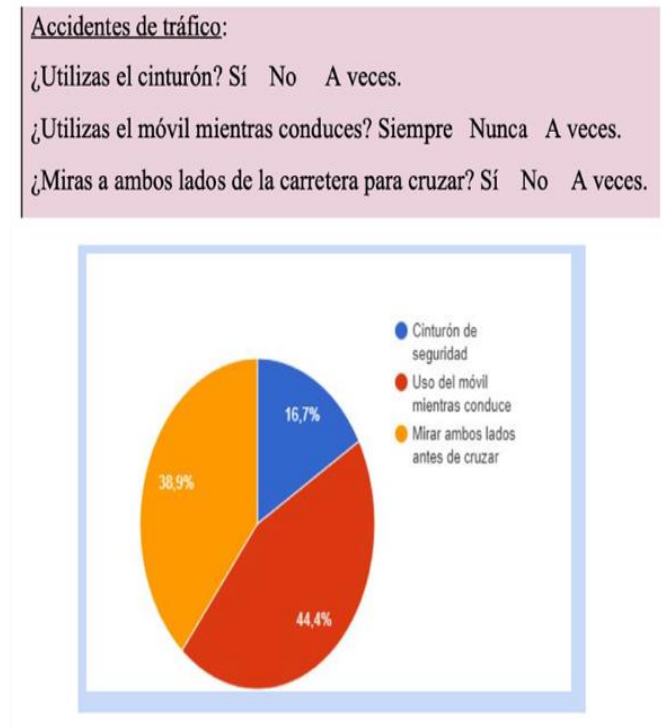

Pregunta y su gráfica incorrecta
En esta segunda actividad han de crear mediante las TICS unas gráficas a partir de análisis de datos de los resultados de las encuestas. En este apartado observamos un error a I hora de representar las encuestas en el gráfico circular, los dos de barras y el de línea. El erro reside en que han representado las preguntas, cuando se debía representar preguntas y la respuestas ya sea en porcentaje 0 en valores. De esta forma se verá la comparación real entr las respuestas seleccionadas por los entrevistados.

Respuesta grupo 101-14 sobre ES-2

Propuesta ES-2 sectores circulares aparece que cada color se corresponde a una de las preguntas realizadas $\mathrm{e}$ relación a los accidentes de tráfico, es decir, un porcentaje se corresponde con el uso de cinturón, otro con el uso del móvil al conducir y otro con mirar a ambos lados antes de cruzar.

Respuesta grupo $102-02$ sobre ES-2

\begin{abstract}
$>$ Sesión 2: Hemos detectado que existe un error matemático en las representaciones de los datos, ya que las gráficas que se han realizado no están correctamente representadas, ni se ha hecho una adecuada comparación de los resultados obtenidos. Ya que al realizar las gráficas presentan datos de preguntas que no tienen relación, ya que son diferentes.
\end{abstract}

Respuesta grupo 102-04 sobre ES-2

Figura 11. Correcciones en informes al gráfico erróneo de la propuesta ES-2.

Fuente: Elaboración propia.

En la Figura 12 mostramos algunas de las actividades planteadas por los estudiantes y su nivel de Reflexión de demanda cognitiva. En ellos se observa que la estadística se muestra como una herramienta que busca que los estudiantes realicen compaciones o extraigan conclusiones con los datos analizados y estos dentro del contexto de trabajo. 


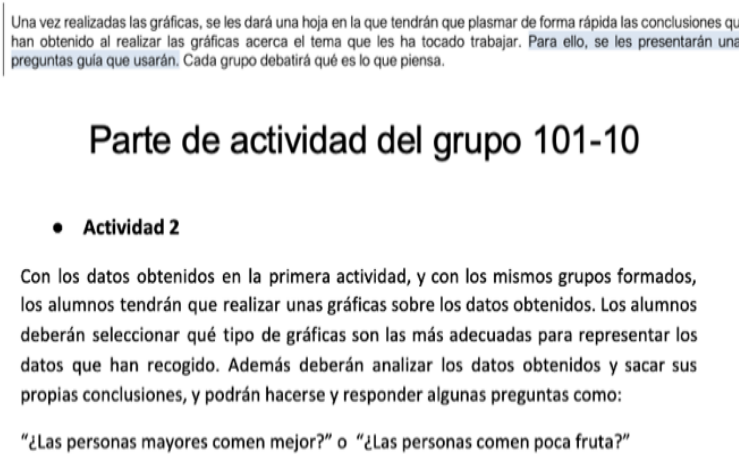

Parte de actividad del grupo 101-10

- Actividad 2

Con los datos obtenidos en la primera actividad, y con los mismos grupos formados, los alumnos tendrán que realizar unas gráficas sobre los datos obtenidos. Los alumnos deberán seleccionar qué tipo de gráficas son las más adecuadas para representar los datos que han recogido. Además deberán analizar los datos obtenidos y sacar sus propias conclusiones, $\mathrm{y}$ podrán hacerse $\mathrm{y}$ responder algunas preguntas como:

"¿Las personas mayores comen mejor?" o "¿las personas comen poca fruta?"

TÍTULO: "El reparto justo". -Por una economía más justa-

OBJETIVO: conocer cómo funciona la cadena del comercio, así como la desigual distribución de los beneficios de la misma (comercio injusto o habitual), asumiendo las interacciones existentes entre las distintas partes de la cadena y tomando conciencia de la necesidad de comunicación entre las mismas para el desarrollo de un comercio más equitativo y justo.

Parte de actividad del grupo 102-13

Para trabajar de manera especifica el ODS de Igualdad de género proponemos recursos como el Atlas mundial de la igualdad de género en la educación, publicado por la UNESCO, donde se encuentran datos, estadisticas y otro tipo de información para analizar el tema a tratar.

Parte de actividad del grupo 101-09

Figura 12. Actividades de la categoría de demanda cognitiva de reflexión.

Fuente: Elaboración propia.

\section{Autenticidad}

La autenticidad es una categoría compleja, por la cantidad de variables que intervienen en su construcción. En ella se analiza que un conjunto de actividades estadísticas, secuenciadas, se desarrollen en el marco de la temática seleccionada, que debe ser cercana a la realidad, y que esta temática no la debe perder de vista a lo largo de todo su desarrollo. Para su análisis es necesario, por tanto, tener la visión del conjunto de actividades y cómo se desarrollan a lo largo de toda la secuencia. En el trabajo de Vásquez y García-Alonso (2020) se cataloga la propuesta ES-2 como una propuesta verosímil, mientras que ES-5 es una propuesta auténtica. La diferencia entre ambas propuestas (Figura 13) radica en los aspectos relacionados con el propósito y la especificidad de los datos, que no se verifican en la propuesta ES-2, mientras que sí se cumplen en la ES-5. 


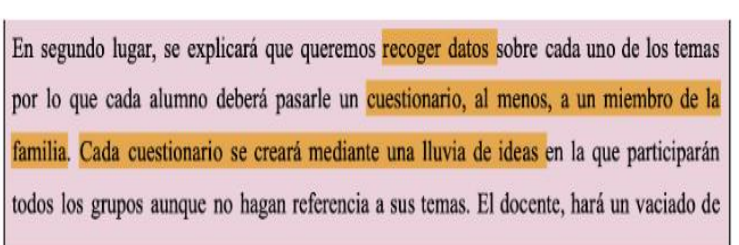

Información: datos reales (Propuesta ES-2)
- ¿Creen que las jornadas deben ser igual de largas para todos los empleos??Por qué?

- Por qué consideran que hay trabajos más envejecidos que otros?

En base a los datos ¿Consideras que hay trabajos más feminizados o masculinizados que otros? ¿Por qué?

¿Crees que estar empleado implica cumplir las características de trabajo decente?

- Aportamos propuestas para mejorar las condiciones de trabajo, hacia un empleo decente, y el crecimiento económico.

Propósito: Explíito y coherente con lo planteado (Propuesta ES-5)

Figura 13. Actividad de mayor demanda cognitiva de cada propuesta.

Fuente: Vásquez y García-Alonso (2020).

Así, para el análisis de la autenticidad partiremos de las nuevas actividades que promueven los diferentes grupos de futuros profesores (Figura 14), y analizamos la propuesta original incluyendo las nuevas actividades que configuran el rediseño de la propuesta original. Dado que ES-5 se ha catalogado como auténtica, realizamos el análisis en el rediseño realizado en la propuesta ES-2, catalogada como verosímil, y estudiamos si las actividades permiten verificar los aspectos de propósito y especificidad de los datos, con lo que se consideraría una propuesta auténtica. Encontramos que 5 equipos (33.3\%) proponen nuevas actividades que hacen que con el nuevo diseño la propuesta ES-2 sea una propuesta auténtica. 


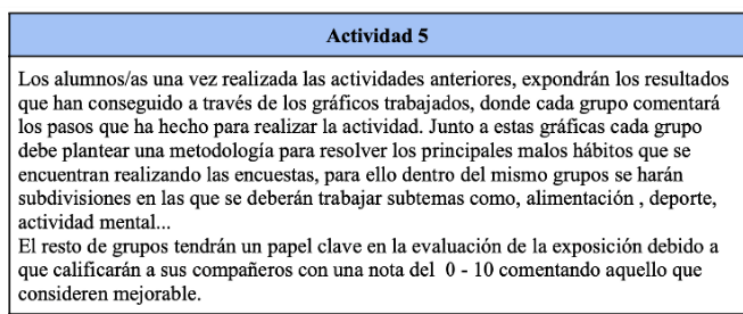

Parte de actividad del grupo 101-02

5. Añadimos preguntas guias en todos los debates relacionadas con el ODS seleccionado para evitar que el debate no se desvie a otro tema y para incentivarlos a dar opiniones, obtener conclusiones y proponer soluciones; ¿Crees qué los hábitos que tiene la población estudiada son buenos o malos? se reflexiona sobre la salud de los ciudadanos y si se debe modificar o no. De esta manera se cumple con ciertos objetivos del ODS, “... puede reflexionar críticamente sobre ellos, incluida la comprensión de la importancia del género en la salud y bienestar.", "EVla alumno/a conoce las estrategias de prevención relevantes para fomentar la salud y el bienestar fisico y mental", "El/a alumno/a es capaz de incluir en sus rutinas diarias conductas que promuevan la salud".

Parte de actividad del grupo 101-10

- Actividad 3

Esta última actividad la realizará la totalidad de la clase, ya que consistirá en una pancarta con la que se fomente una vida sana. Será interesante que los mensajes que se coloquen en la pancarta, partan de los datos obtenidos en las encuestas. Asi, por ejemplo, si con los datos obtenidos concluimos que se come menos fruta de la que se debería, encaminariamos nuestros mensajes a este punto.

Parte de actividad del grupo 101-12

\section{3o sesión}

Para finalizar la situación de aprendizaje, como producto final, cada grupo realizará una presentación utilizando PowerPoint, Genially o Canva. Para estas presentaciones el docente dará previamente unas "pildoras formativas" sobre el uso de dichos recursos TIC. A su vez, durante la realización de las mismas, el docente facilitará su ayuda al grupo que lo necesite.

Además, con el objetivo de ir mejorando poco a poco los resultados, se plantearán retos semanales relacionados con las preguntas planteadas. Por ejemplo, en caso de no consumir el número de piezas de fruta recomendado, se planteará como reto llevar durante toda la semana una fruta para desayunar. De este modo, el alumnado se involucrará en el cambio de sus hábitos de una forma más directa.

Parte de actividad del grupo 102-02

Actividad: iVamos al mercadillo

Con esta actividad, pretendemos que sirva de nexo de unión con la actividad anterior, después de que el alumnado haya investigado sobre los hábitos de vida saludable. Llevaremos a la clase de visita al Mercadillo del Agricultor, en la Matanza.

A lo largo de los diferentes puestos, los niños y niñas deberán de identificar los alimentos saludables que más les guste y escribirlos en una hoja.

Después de la visita, por pequeños grupos de trabajo, tendrán que escribir un menú de un día con platos saludables con los alimentos elegidos.

Por último, una vez obtenida toda la información, realizarán una exposición relacionando los datos obtenidos con las medidas para mejorar los hábitos de vida saludables, es decir, propondrán una serie de medidas para llevar una vida más saludable. Y añadirán la propuesta de menú con los alimentos seleccionados.

Parte de actividad del grupo 102-10

Figura 14. Ejemplos de actividades que incorporadas a la propuesta ES-2 la hacen auténtica.

Fuente: Elaboración propia.

\section{CONCLUSIONES}

A través de este análisis hemos estudiado cómo futuros profesores de Educación Primaria se enfrentan al análisis y al rediseño de una tarea que fomenta el aprendizaje de estadística en estudiantes de Educación Primaria con foco en sostenibilidad. En dicho análisis hemos identificado los aspectos en los que han incidido los futuros profesores y de qué manera estos aspectos han permitido que ahonden en el desarrollo de un conocimiento estadístico y sobre la sostenibilidad.

Para el análisis hemos utilizado un instrumento compuesto por cuatro características: contexto, nivel de articulación, demanda cognitiva y autenticidad. Este 
instrumento ha sido capaz de poner de relieve de qué manera, cuando se enfrentan a dos tareas diferentes, los estudiantes son capaces de proponer nuevas actividades que busquen centrar la tarea original de manera más adecuada en el ODS seleccionado.

Es relevante que una de las tareas que analizaron y debían rediseñar es clasificada como auténtica. Nos referimos a la tarea ES-5, sobre la que 5 grupos consideraron que se encontraba bien enfocado el contexto, 7 grupos indicaron que el ODS se encontraba bien articulado y para la que 6 grupos ofrecieron actividades de demanda cognitiva de la categoría de Reflexión. En cambio, para la tarea categorizada como verosímil (ES-2), ninguno de los grupos de estudiantes consideró el contexto adecuado, 9 grupos consideró que el ODS no se encontraba bien articulado en la propuesta y sólo 4 grupos ofrecieron actividades cuya demanda cognitiva fuese de Reflexión. Consideramos estas diferencias como un indicio de que las tareas auténticas son perceptibles por los propios docentes en formación, aún cuando no han recibido formación al respecto. Y, además, contar con una tarea de estas características son modelos que se incorporan a su formación, en este caso, con alta demanda cognitiva y con foco en sostenibilidad.

Atendiendo a la autenticidad, hemos podido observar que un tercio de los grupos que trabajaron con la tarea verosímil fueron capaces de incorporar actividades que permitieron el avance de la tarea inicial a una tarea auténtica. Aunque este resultado puede parecer poco relevante, creemos que con una pequeña formación se podría lograr un mayor avance en este sentido.

Por otro lado, sí nos parece muy poco significativo el número de grupos de estudiantes que fueron capaces de detectar errores en la tarea estadística. Esto pone de manifiesto que es necesario profundizar en el conocimiento estadístico de los futuros profesores para que sean capaces de detectar y corregir los posibles errores en tareas, tanto en aquellas que están listas para llevar al aula como las que puedan plantear sus estudiantes. 


\section{Universidade Federal da Grande Dourados}

A modo de conclusión, los futuros profesores necesitan profundizar en el conocimiento estadístico que subyace en las tareas propuestas para llevar al aula. Pero, cuando analizan o rediseñan actividades estadísticas con foco en sostenibilidad, los docentes sin formación específica previa son capaces de promover aspectos que redirigen la mirada hacia tareas potentes que aglutinen los contextos de los ODS e incorporen tanto una alta demanda cognitiva como una articulación adecuada de estos contextos a lo largo de toda la propuesta. Además, las tareas así construidas se pueden redirigir de forma que verifiquen las características de la autenticidad, lo que permitirá desarrollar en el aula una enseñanza dirigida a conseguir la EDS.

Finalmente, no cabe duda de que la Educación para el Desarrollo Sostenible es un reto que la UNESCO ha lanzado a toda la sociedad y a la comunidad educativa en particular. Esto implica, no sólo cambios en la selección de los contenidos a enseñar, sino también en los recursos que utilizamos para la enseñanza. La Educación Estocástica es un conocimiento que se alinea perfectamente con una EDS, pero es necesario pensar cómo articularlos para lograr un desarrollo efectivo de los ODS en el aula. Las categorías de análisis utilizadas en este trabajo no sólo permiten describir de qué forma los recursos de Educación Estocástica promueven una EDS, sino que, además, señalan y facilitan los aspectos del diseño de tareas concretas a incorporar para promover una EDS efectiva y una enseñanza contextualizada, dirigida a desarrollar alfabetizados en sostenibilidad, que tomen conciencia de las problemáticas de índole social, económico y medioambiental para actuar y avanzar hacía un mundo más sostenible (UNESCO, 2017).

\section{AGRADECIMIENTOS}

Trabajo realizado en el marco del proyecto FONDECYT № 1200356 financiado por la Agencia Nacional de Investigación y Desarrollo del Gobierno de Chile. 


\section{Universidade Federal da Grande Dourados}

\section{REFERENCIAS}

Batanero, C. (2019). Thirty years of stochastics education research: Reflections and Challenges. In J. M. Contreras, M. M. Gea, M. M. López-Martín \& E. Molina-Portillo (Eds.), Actas del Tercer Congreso Internacional Virtual de Educación Estadística (pp. 1-15). Retrieved from https://www.ugr.es/ fam126/civeest/ponencias/batanero ing.pdf

Batanero, C. \& Borovcnik, M. (2016). Statistics and probability in high school. Rotterdamn, The Netherlands: Sense Publishers.

Ben-Zvi, D., \& Garfield, J. (2004). Statistical literacy, reasoning, and thinking: Goals, definitions, and challenges. In D. Ben-Zvi \& J. Garfield (Eds.), The challenge of developing statistical literacy, reasoning and thinking (pp. 315). The Netherlands: Kluwer Academic Publishers.

https://doi.org/10.1007/1-4020-2278-6 1

Ben-Zvi, D., Makar, K. \& Garfield, J. (Eds.). (2018). International Handbook of Research in Statistics Education. Cham: Springer. DOI: 10.1007/978-94010-0462-6

Calero, M., Mayoral, O., Ull, A., \& Vilches, A. (2019). La educación para la sostenibilidad en la formación del profesorado de ciencias experimentales en Secundaria. Enseñanza de las Ciencias, 37(1), 157-175. https://doi.org/10.5565/rev/ensciencias.2605 


\section{Universidade Federal da Grande Dourados}

Chamoso, J.M., \& Cáceres, M.J. (2018). Propuesta de tareas matemáticas en contextos reales de estudiantes para maestro. Cuadernos de Investigación y Formación en Educación Matemática, 17, 83-94.

Cobb, G., \& Moore, D. (1997). "Mathematics, Statistics, and Teaching". American Mathematical Monthly, 104 (9), 801-823.

https://doi.org/10.1080/00029890.1997.11990723

Dahl, T. (2019). Prepared to Teach for Sustainable Development? Student Teachers' Beliefs in Their Ability to Teach for Sustainable Development.

Sustainability, 11, 1993-2103.

Engel, J. (2019). Cultura estadística y sociedad. En J. M. Contreras, M. M. Gea, M. M. López-Martín y E. Molina-Portillo (Eds.), Actas del Tercer Congreso Internacional Virtual de Educación Estadística (pp. 1-18). Granada: Grupo FQM-126. www.ugr.es/local/fqm126/civeest.html

Frankenstein, M. (2009). Developing a critical mathematical numeracy through real real-life word problems. In L. Verschaffel, B. Greer, W. Van Dooran, \& S. Mukhopadhyay (Eds.), Words and worlds: Modeling verbal descriptions of situations (pp. 111-130). Rotterdam, The Netherlands: Sense Publishers.

Gal, I. (2002). Adults' Statistical literacy: meanings, components, responsibilities. International Statistical Review, Nederlandn, n. 70, p. 1-25.

Gal, I. (2005). Towards 'probability literacy' for all citizens. In G. Jones (ed.), Exploring probability in school: Challenges for teaching and learning (pp. 43-71). Kluwer Academic Publishers. 


\section{Universidade Federal da Grande Dourados}

Gal, I. (2019). Understanding statistical literacy: About knowledge of contexts and models. En J. M. Contreras, M. M. Gea, M. M. López-Martín y E. MolinaPortillo (Eds.), Actas del Tercer Congreso International Virtual de Educación Estadística. www.ugr.es/local/fqm126/civeest.html

Garfield, J. (2002). The challenge of developing statistical reasoning. Journal of Statistics Education, 10(3).

\section{https://doi.org/10.1080/10691898.2002.11910676}

Gattuso, L., \& Ottaviani, M. G. (2011). Complementing mathematical thinking and statistical thinking in school mathematics. In C. Batanero, G. Burrill, \& C. Reading (Eds.), Teaching statistics in school mathematics-challenges for teaching and teacher education: A joint ICMI/IASE study (pp. 121-132). Dordrecht, Netherlands: Springer. https://doi.org/10.1007/978-94-007$\underline{1131-0}$

Lesser, L.M. (2007). Critical values and transforming data: Teaching statistics with social justice. Journal of Statistics Education, 15(1), 1-21. https://doi.org/10.1080/10691898.2007

McMillan, J.H. \& Schumacher, S. (2001). Research in Education. A conceptual introduction $5^{\text {th }}$ edition: New Jersey, USA: Pearson.

National Council of Teachers of Mathematics [NCTM] (2014). Principles to Actions: Ensuring Mathematical Success for All. Reston, Va.: NCTM. Reston, Va.: The National Council of Teachers of Mathematics.

Paredes, S., Cáceres, M.J., Diego-Mantecón, J.M., Blanco, T.F. \& Chamoso, J.M. (2020). Creating realistic mathematics task involving authenticity, cognitive 


\section{Universidade Federal da Grande Dourados}

domains, and openness characteristics: A study with pre-service teachers. Sustainability, 12, 9656, https://doi:10.3390/su12229656

Pfannkuch, M., \& Wild, C. (2004). Towards an understanding of statistical thinking. In J. B. Garfield \& D. Ben-Zvi (Eds.), The challenge of developing statistical literacy, reasoning and thinking (pp. 17-46). Dordrecht, Netherlands:

Springer. https://doi.org/10.1007/1-4020-2278-6 2

Stemler, S. (2001). An overview of content analysis. Practical Assessment, Research \& Evaluation, 7(17), 1-6. https://doi.org/10.7275/z6fm-2e34

Stibbe, A. (2014). The Handbook of Sustainability Literacy: Skills for a Changing World. Green Books.

Uitto, A. \& Saloranta, S. (2017). Subject Teachers as Educators for Sustainability: A Survey Study. Education Sciences, 7 (1), 8-26.

UNESCO. (2015). Transforming our world: the 2030 Agenda for Sustainable Development. Retrieved from https://www.un.org/ga/search/view doc.asp?symbol=A/RES/70/1\&Lang=E

UNESCO. (2017). Education for Sustainable Development Goals: learning objectives. Retrieved from https://unesdoc.unesco.org/ark:/48223/pf0000247444

Usiskin, Z. (2014). On the relationships between statistics and other subjects in the K-12 curriculum. In K. Makar, B. de Sousa, y R. Gould (Eds.), Sustainability in Statistics Education. Proceedings of the Ninth International Conference on Teaching Statistics (ICOTS-9), Flagstaff, AZ. Voorburg, The Netherlands: International Statistical Institute. 
Vásquez, C. (2020). Educación estocástica: una herramienta para formar ciudadanos de sostenibilidad. Matemáticas, Educación y Sociedad 3(2), 1 20.

Vásquez, C. (2021). Comprensión y Uso Docente de Gráficos Estadísticos por Futuros Profesores para Promover Competencias para la Sostenibilidad. PARADIGMA, 41(e1), 165-190.

https://doi.org/10.37618/PARADIGMA.1011-2251.2021.p165-190.id1022

Vásquez, C. \& Alsina, Á. (2021). Conectando Educación Estadística y Educación para la Sostenibilidad: un marco para promover el desarrollo sostenible en la formación del profesorado. En Sola, T., Alonso, S., Fernández, M. G. y De La Cruz, J. C. (Eds.), Estudios sobre Innovación e Investigación Educativa (pp. 973-985). Madrid, España. Editorial DYKINSON, S. L. ISBN: 978-84-1377-303-2

Vásquez, C., \& García-Alonso, I. (2020). La educación estadística para el Desarrollo Sostenible en la Formación del Profesorado. Profesorado. Currículum y formación del profesorado, 24(3), 125-147. https://doi.org/10.30827/profesorado.v24i3.15214

Vásquez, C., García-Alonso, I., Seckel, M.J. \& Alsina, Á. (2021). Education for Sustainable Development in Primary Education Textbooks-An Educational Approach from Statistical and Probabilistic Literacy. Sustainability, 13(6), 3115. https://doi.org/10.3390/su13063115 


\section{Universidade Federal da Grande Dourados}

Vásquez, C., Seckel, M. J. \& Alsina, A. (2020). Sistema de creencias de los futuros maestros sobre Educación para el Desarrollo Sostenible en la clase de matemática. Revista Uniciencia, v. 34, n. 2, 16-30.

Vilches, A. \& Gil, D. (2012). La educación para la sostenibilidad: el reto de la formación del profesorado. Profesorado, Revista de currículum y formación de profesorado, 16(2), 25-43.

Wild, C.J., Utts, J.M. \& Horton, N.J. (2018). What is statistics? En D. Ben-Zvi, K. Makar y J. Garfield (Eds.), International Handbook of Research in Statistics Education (pp. 5-36). Cham, Suiza: Springer.

Zapata-Cardona, L. (2018). Enseñanza de la estadística desde una perspectiva crítica. Yupana, 10, 30-41. https://doi.org/10.14409/yu.v0i10.7695 